\title{
Redefining Gravity: Field versus Flow
}

\author{
Mehmet Bora Cilek ${ }^{1}$ \\ ${ }^{1}$ PFR Varyap Meridian E148, 34746 Turkey \\ Correspondence: M. Bora Cilek, PFR Varyap Meridian E148, 34746 Turkey. E-mail: gravity@superonline.com
}

Received: March 16, 2017

Accepted: March 26, 2017 Online Published: March 31, 2017

doi:10.5539/apr.v9n2p87

URL: https://doi.org/10.5539/apr.v9n2p87

\begin{abstract}
General Theory of Relativity constitutes the framework for our understanding of the universe, with an emphasis on gravity. Many of Einstein's predictions have been verified experimentally but General and Special Theories of Relativity contain several anomalies and paradoxes, yet to be answered. Also, there are serious conflicts with Quantum Mechanics; gravity being the weakest and least understood force, is a major problem.

Supported by clear experimental evidence, it is theorised that gravity is not a field or spacetime curvature effect, but rather has a flow mechanism. This is not an alternative theory of gravity with an alternative metric. Established laws and equations from Newton and Einstein are essentially left unchanged. However, spacetime curvature is replaced with flow, producing a refined and compatible theory.
\end{abstract}

Keywords: Gravity; Relativity; Fluid Universe; Gravitation; Interferometry; Aether; Vertical Michelson-Morley Experiment

\section{Introduction}

The question of gravity was always a major concern for science throughout the history. First detailed analysis was carried out by Isaac Newton in 17th century, exposing mathematical relations governing gravitation and motion (Newton, 1687). However Newton himself accepted that he did not know what exactly caused gravity and was puzzled by the possibility of action/force over a distance. More than two centuries later, Einstein opened a new window on the subject by introducing Relativity and the concept of spacetime (Einstein, 1915).

Relativity now enjoys an unprecedented popularity with the verification of its various predictions. But we also know that there are several problems associated with it (Note 1), puzzling scientists for decades. Incompatibility with Quantum Mechanics is another concern, thanks mainly to gravity.

Convinced by the Equivalence Principle, Einstein based General Relativity upon the concept of curved spacetime, which he believed and proposed to be an exclusive solution. This describes gravity not as a force but as a consequence of the uneven distribution of mass/energy, resulting in gravitational time dilation. This approach may verify orbital motion, the bending of light near a massive star or even the path of an apple falling toward earth; but it cannot explain 'why' the apple falls down from the tree.

Consequently, after more than a century with Relativity, we are still far from fully understanding gravity, let alone manipulating it. This is not the case with any other major theory.

It is hereby suggested that the problem with General Theory of Relativity lies within the concept of spacetime, which introduces unnecessary complications by ignoring a much simpler solution. Through experimental evidence, it will be shown that flow model not only satisfies the equivelance principle but also produces a simpler and better framework for gravity and Relativity in general.

Theoretical discussion presented in this paper is a result of extraordinary empirical data, which will be detailed in the next section.

\section{Methods and Experiment}

The Theory is based on a modified version of the Michelson-Morley experiment (Michelson \& Morley, 1887), originally conducted in 1887. This and subsequent versions by different teams employed the rotation of the arms of a Michelson Interferometer in the horizontal plane, parallel to earth's surface. This would measure the speed of the claimed 'aether flow' as the earth orbits the sun (expected to be at least $30 \mathrm{~km} / \mathrm{s}$ ). They all produced null results, apparently disproving aether and showing that light does not need a medium to propagate in. 
The modification presented here involves the rotation of one the arms of the interferometer in the vertical plane, to investigate the effect of gravity on light that has a perpendicular path to earth's surface.

This kind of vertical setup introduces serious problems, like the deformation of the frame and misalignment of components under their own weight. When the wavelength of light is considered, it is obvious that all such effects due to gravity and rotation must be minimized to produce meaningful results, with extreme attention to the quality and assembly of parts. With these considerations it was decided to conduct the experiment under water, using a cylindrical frame with a rigid structure. The material of choice was HDPE (High Density Polyethylene) (Note 2) which has a very high strength-to-density ratio. It neither sinks nor is buoyed up due to its almost identical density $\left(0.98 \mathrm{~g} / \mathrm{cm}^{3}\right)$ to water, practically creating a weightless environment for the interferometer mounted inside. The cylindrical frame can be rotated underwater without difficulty. Its inner diameter and height were 140 and 160 centimeters respectively.

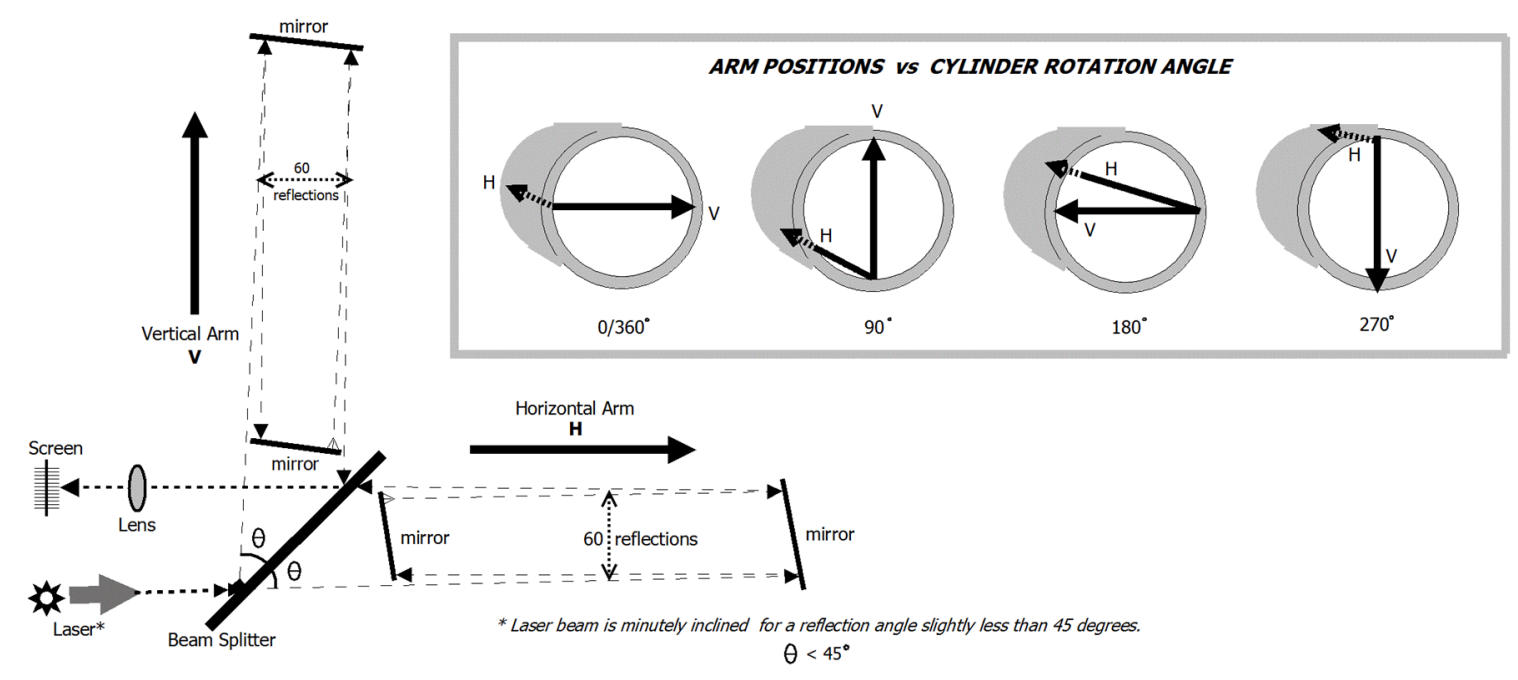

Figure 1. Schematics of the modified Michelson Interferometer and its mounting in the cylindrical frame. For simplicity, some components are omitted and distances/angles may not be in correct scale

As can be seen in figure 1, light is reflected 60 times inside interferometer arms via slightly angled mirrors, to obtain an equivalent arm length of 72 meters. The analysis section which comes next describes how this path length is predetermined. A $532 \mathrm{~nm}$, narrow beam-far range laser module with exteremely low divergence angle and collimation was used throughout the experiments. Beam width was less than $1 \mathrm{~mm}$ and reflected beams were approximately $2.5 \mathrm{~mm}$ apart. In calculations, speed of light in water is taken as $225,000 \mathrm{~km} / \mathrm{s}$ which gives a wavelength of around 400 nanometers for the green laser used.

Current theory states that light, being an electromagnetic wave, does not need a medium to propagate and has a constant speed at every direction. Thus, there should be no shift in the interference pattern regardless of how you rotate the setup, in horizontal or any vertical plane. In the case of upward propagation, there must be a redshift in frequency due to gravity, only to be canceled out by an equal blueshift while coming down back, producing no shift again.

\section{Results}

Measurements were taken on 2 different days, at $7 \mathrm{am}$ and $7 \mathrm{pm}$ (before dawn and after dusk ) in December 2016. Ambient temperature was the same as the pool temperature, $24{ }^{\circ} \mathrm{C}$. First, the arms were rotated horizontally to repeat the classical Michelson-Morley experiment. No significant fringe shifts were observed. This also served as a quick check for systematic and calibration errors before each run. Then one of the arms (vertical arm - V) was erected slowly by rotating the cylinder counterclockwise around its central axis at a rate of 30 degrees per minute, while the other (horizontal arm - H) stayed parallel to earth. It was observed that the fringe pattern shifted during this rotation, being maximised at 90 and 270 degrees, that is when one arm (V) is perpendicular to earth's surface. Furthermore the shift was always in the same direction and indicated that light is delayed in the vertical arm 
whether it is pointing up or down (this also served as a test to check that fringe shift is not related to deformation of the setup).

Results are summarized below.

Table 1. Average number of fringe shifts (in wavelengths, over 8 runs) versus rotation angle, for an effective arm length of 72 meters with a $532 \mathrm{~nm}(\lambda \approx 400 \mathrm{~nm}$ in water) laser

\begin{tabular}{lllllllll}
\hline Rotation Angle (degrees) & 0 & 45 & 90 & 135 & 180 & 225 & 270 & 315 \\
\hline Mean Fringe Shift (wavelengths) & $0(\mathrm{rf})$ & 0.55 & 0.84 & 0.58 & 0.02 & 0.56 & 0.88 & 0.56 \\
\hline
\end{tabular}

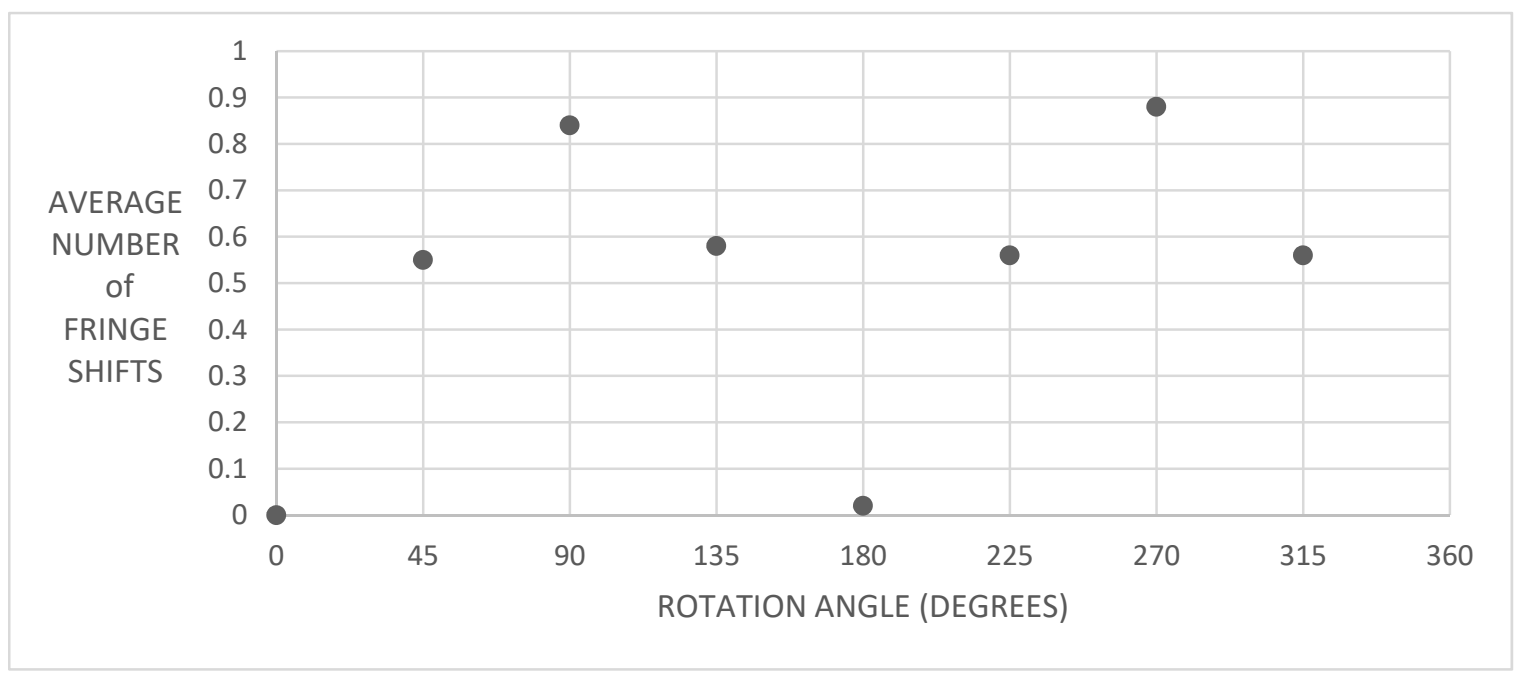

Figure 1. Average fringe shift (in number of wavelengths) plotted against rotation angle

As seen from the Figure 1, data has the characteristic of ' $|\sin \mathrm{x}|$ ' function, with slightly off (lower) readings at $45,135,225$ and 315 degrees.

From the results summarized above, it is inevitable to deduce that light travels in a different manner vertically, than it does horizontally. Without breaking accepted laws of physics (speed of light is constant, and light would be bent toward the gravitating mass) this exclusively implies that space flows into earth or in other words, earth sucks the space around it. Furthermore, detailed analysis of data in table 2 indicates that the speed of this accelerated flow approximates $\sqrt{2 G M / r}$, escape velocity on earth's surface, as predicted by the discussion in the next section.

Observations at 90 and 270 degrees are summarized below, that is when one arm is perpendicular to earth :

Table 2. Recorded number of fringe shifts and implied flow speeds at 90 and 270 degrees (perpendicular positions)

\begin{tabular}{lllll}
\hline day & time & angle (degrees) & \# of fringe shifts (wavelengths) & implied flow speed $(\mathrm{km} / \mathrm{s})$ \\
\hline 1 & $7 \mathrm{am}$ & 90 & 0.84 & 10.5 \\
1 & $7 \mathrm{am}$ & 270 & 0.76 & 9.5 \\
1 & $7 \mathrm{pm}$ & 90 & 0.80 & 10 \\
1 & $7 \mathrm{pm}$ & 270 & 0.92 & 11.5 \\
2 & $7 \mathrm{am}$ & 90 & 0.88 & 11 \\
2 & $7 \mathrm{am}$ & 270 & 0.96 & 12 \\
2 & $7 \mathrm{pm}$ & 90 & 0.84 & 10.5 \\
2 & $7 \mathrm{pm}$ & 270 & 0.88 & 11 \\
\hline
\end{tabular}


Observational sensitivity was about $1 / 25$ th of a wavelength (4\%). Average fringe shift was 0.86 wavelengths, corresponding to a flow speed of $10.75 \mathrm{~km} / \mathrm{s}$. All shifts were in the same direction. For calculating implied flow speeds at 90 and 270 degrees, the equation

$$
\text { number of fringe shifts (in } \lambda \text { 's) }=2 \mathrm{~L}\left(\frac{c^{2}}{c^{2}-v^{2}}-1\right) / \lambda \approx 2 \mathrm{Lv}^{2} / \lambda \mathrm{c}^{2}
$$

can safely be used ( $\mathrm{L}$ is the total effective arm length).

Another point for consideration is that despite the earth moves through space as it orbits the sun, there are no fringe shifts when the arms are horizontal (just like the original Michelson-Morley experiment, no 'absolute motion' was detected). This strongly suggests that any spatially isolated lump of matter which is in free fall, creates its own reference frame that flows inward from every direction and gets carried along during motion.

\section{Analysis and Discussion}

Supported by experimental results in the previous section, it is theorised that matter does not curve/bend the surrounding space but rather sucks it in toward the center of mass, with a flow speed of $\sqrt{2 G M / r}$ at any

given distance $r$ from its center of mass (idealized case for a simple shaped body with mass $\mathrm{M}, \mathrm{r}>>$ maximum structural radius of the body; $\mathrm{G} \approx 6.67 \times 10^{-11} \mathrm{~m}^{3} \mathrm{~kg}^{-1} \mathrm{~s}^{-2}$ is the gravitational constant and flow speed is in $\mathrm{m} / \mathrm{s}$ ).

In other words, gravity is the result of the accelerated flow of space into the gravitating mass, with a resultant force on all other bodies (due to relative acceleration with respect to the reference frame, $\mathrm{F}=\mathrm{ma}$ ). The structure and properties of (empty) space (Note 3) will not be discussed here, however once again, it is crucial to consider this as the reference frame flowing toward the gravitating body.

Despite experimental results suggesting a flow speed reminiscent of earth's escape velocity $(11.2 \mathrm{~km} / \mathrm{s})$, it would be appropriate to determine the formulation for flow using conventional physics, before assigning any meaning to the measurements (Note 4). This was also necessary to determine the correct scale for the experiment, i.e. the effective length of interferometer arms.

\subsection{Derivation from Classical Mechanics}

Flow speed at any given coordinate is the main parameter to be determined. Take the hypothetical case of a large gravitating body with mass $\mathrm{M}$ and an (almost) infinitely remote/small particle of mass $\mathrm{m}$, both at rest to begin with. According to classical mechanics; starting with a kinetic energy of zero, ' $\mathrm{m}$ ' will gradually accelerate and attain a kinetic energy of $G M m / r$ and a speed of $\sqrt{2 G M / r}$, at any distance $r$ to the larger mass. In the flow model for gravitation however, our infinitesimal particle is stationary with respect to the reference frame while frame itself (space) flows toward the gravitating body, thus their speeds will be identical. This gives us

$$
\mathrm{V}_{\text {flow }}=\sqrt{\frac{2 G M}{r}}
$$

at distance $r$. As mentioned above, this is equal to the escape velocity in classical physics.

In generalized vector form, flow velocity (with respect to mass $\mathrm{M}$ ) at vector position $\mathbf{r}$ will be ;

$$
\mathbf{V}_{\mathrm{M}}(\mathbf{r})=-\sqrt{\frac{2 G M}{|\mathbf{r}|}} \hat{\mathbf{r}}
$$

where $\hat{r}$ is the unit vector from the center of mass (of ' $M$ ') to the given position.

For a quick reality check, we should be able to derive gravitational acceleration $\mathbf{g}$, which is the key to Newton's Law of Gravitation;

$$
\mathbf{g}=\frac{d \mathbf{V}}{d t}=\frac{d \mathbf{V}}{d \mathbf{r}} \frac{d \mathbf{r}}{d t}=\frac{d \mathbf{V}}{d \mathbf{r}} \mathbf{v}=-\frac{\sqrt{2 G M}}{2 r^{3 / 2}} \frac{\sqrt{2 G M}}{\sqrt{r}} \hat{\mathbf{r}}=-\frac{G M}{r^{2}} \hat{\mathbf{r}}
$$

where there are no surprises.

A flow speed proportional to $1 / \sqrt{r}$ is also meaningful from the geometrical viewpoint. Volumetric flow rate must be constant through all cocentric spherical surfaces around the center of mass, since space should neither be created nor destroyed. Flow speed and three spatial dimensions each get concentrated to higher density in proportion to $\sqrt{r}$, multiplying to $r^{2}$, which perfectly compensates for the change in spherical areas (in proportion to $1 / r^{2}$ ) leading to the gravitating body. "What happens to space that is sucked in by mass" is a subject for pure speculation at this stage and will not be discussed here. But it might be a useful analogy to think that as 'fuel', required for the stability of matter. 


\section{Conclusion and Remarks}

Although each would be the subject of another paper on its own, it would be appropriate to mention the implications of the theory for some of the controversial topics in physics.

\subsection{Inertia and Motion}

As suggested by flow (and also by Relativity), the universe is dynamic and almost everything is in relative motion. One of the main conclusions of this article is that every isolated object/matter in free fall creates its own reference frame that flows toward its center of mass. This reference frame is carried along during motion, as the experiment confirms. In case of acceleration, flow pattern is momentarily disrupted by compressive and decompressive adjustment waves, in the same and opposite directions with acceleration respectively. These travel at the speed of light and correspond to the present concept of gravitational waves. Only during this temporary readjustment the accelerated body will experience structural stress (and time dilation), until its reference frame corrects itself and flow pattern reaches equilibrium again with uniform motion. Energy required for acceleration is simply transferred to and stored in the flow, showing up as kinetic energy in the equations of classical physics. What we call 'inertia' is simply this flow pattern's resistance to change, during acceleration.

It is also interesting to note that uniform motion should actually not be possible according to Relativity, since every moving body will continuously emit gravitational waves and dissipate energy regardless of its speed and acceleration. This is because every disturbance in spacetime will produce gravitational waves in Einstein's spacetime curvature model. This is not the case with flow, where gravitational waves are emitted only during acceleration.

\subsection{Galaxy Rotation Curves}

Flow Model's solution for the dark matter problem is based on the 'frame dragging' effect, which was experimentally confirmed in the last decade. We know that at the center of every significant galaxy lies a supermassive blackhole, spinning extremely fast. This spin also drags space with an angular velocity inversely proportional to the cube of the distance, converging to the speed of light near the event horizon. In the flow model, this introduces a tangential component for the gravitational acceleration vector, which gets comparable and even surpasses the radial component, as one gets closer to the blackhole. (An anology can be made with water swirling in a sink). This results in a smaller (radial) gravitational effect and thus less than expected orbital speed for nearby stars, as opposed to an (almost) pure radial vector and normal orbital speeds for stars at the outskirts of the galaxy. Relevant equations can be derived by adapting the flow vector to Kerr Metric. This approach has important consequences:

1) Central blackholes (galactic centers) are much heavier than previously calculated.

2) Orbital speeds of outer stars (right end of the rotation curve) give a better indication of galactic center's mass.

3) Dark matter is not necessary to explain flat galaxy rotation curves and extra gravitational lensing.

Same reasoning is also valid for galaxy clusters. Please note that the above summarized approach can also be applied to General Relativity via skewed/warped gravitational field lines due to frame dragging, albeit with limited effects. Also, it is very likely that frame dragging bears the main responsibility for the characteristic shape of spiral galaxies, reminiscent of a whirlpool.

\section{Consequences}

Beside answering major problems in cosmology like flat galaxy rotation curves and the elusive "dark matter", Flow Model will pave the way for the 'Unification of Forces' and reconciliation with Quantum Mechanics. Key concepts of Relativity (like time-dilation, mass-energy equivalence, gravitational redshift, blackholes, gravitational waves, etc..) and even the 'meaning of neutrinos' will be revised according to the flow model, bringing new insight into different fields. Also, speed of light 'c' will have to be rephrased not as an absolute/universal limit but rather an apparent/local one.

On technology side, an important prospect will be the ability to manipulate gravity resulting in a new generation of propulsion methods, gravity shielding, artificial gravity without spin and so forth. Physical-electromagnetic shielding and cloaking, inertial isolation, unobstructed communications via gravitational waves will be among other benefits. It is easy to predict that revolutionary changes in almost every area; from space travel to drone technology, daily commuting to environmental protection, propulsion systems to communications are very much likely.

There are numerous alternative theories of gravity at present and some are more complicated than the General Theory of Relativity itself. Altough 'suction' is commonly used as an anology (like in describing blackholes) and 
was implicitly assumed by theories like the 'Big Crunch', it has never been considered as a viable alternative. The author of this paper is neither the first to propose a flow model for gravity, nor the only one who conducted a customized Michelson-Morley experiment. However this is the first time that flow concept is tested through a well designed experiment with correct scale and sensitivity. Consequently, this is the first time flow becomes a serious alternative to Einstein's spacetime curvature, with a precise and simple theoretical framework.

\section{References}

Newton, I. (1687). Philosophiae naturalis principia mathematica, 3. London.

Einstein, A. (1915). Die feldgleichungen der gravitation. The Field Equations of Gravitation. Berlin.

Michelson, A. A. \& Morley, E. W. (1887). On the Relative Motion of the Earth and the Luminiferous Ether. AJS, 34, 333-345.

\section{Notes}

Note 1. Need for the elusive dark matter to explain flat galaxy rotation curves, anomalies in blackhole physics, inconsistencies in subtheories like 'Big Bang' and 'Inflation', and the question of dark energy are just some of the problems in General Theory of Relativity.

Note 2. This material is commonly used for underground pipes of infrastructure, where durability is required under tough conditions.

Note 3. It must be noted that several alternative theories have been proposed in the past about the mechanics of gravitation and the structure of space, questioning what vacuum really is, with the provision that empty space may indeed not be empty. Luminiferous Aether was arguably the most popular of these theories (even became mainstream for sometime) but was deemed invalid by the Michaelson-Morley experiment in 1887. With new theoretical and experimental suggestions in past decades however, ideas such as "superfluid universe" and models like "cosmic molasses" were brought into attention again. It is also interesting to note that General Theory of Relativity itself assumes that space (not only spacetime-see frame dragging) is flexible/malleable; it can be bent, twisted, and warped.

Note 4. Once flow is considered as a viable model, one can easily see that its key metric 'flow speed' should be equal to the 'escape velocity' at any given position, to yield the correct gravitational acceleration in consistence with established physics. Having noted this, standard methodology follows for a complete derivation from scratch.

\section{Copyrights}

Copyright for this article is retained by the author(s), with first publication rights granted to the journal.

This is an open-access article distributed under the terms and conditions of the Creative Commons Attribution license (http://creativecommons.org/licenses/by/4.0/). 